\title{
Asymmetric expression of homoeologous genes contributes to dietary adaption of an allodiploid hybrid fish derived from Megalobrama amblycephala () $\times$ Culter alburnus ( $(\hat{)})$
}

Wuhui $\mathrm{Li}^{1,2+}$, Shi Wang ${ }^{1 \dagger}$, Jie Hu${ }^{2 \dagger}$, Chenchen Tang ${ }^{1}$, Chang Wu${ }^{1}$, Junmei Liu', Li Ren ${ }^{1}$, Chengfei Sun ${ }^{2}$, Junjian Dong ${ }^{2}$, Shaojun Liu $^{1^{*}}$ (I) and Xing Ye ${ }^{2^{*}}$

\begin{abstract}
Background: Hybridization, which can quickly merge two or more divergent genomes and form new allopolyploids, is an important technique in fish genetic breeding. However, the merged subgenomes must adjust and coexist with one another in a single nucleus, which may cause subgenome interaction and dominance at the gene expression level and has been observed in some allopolyploid plants. In our previous studies, newly formed

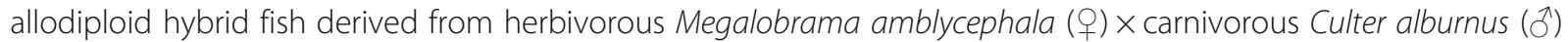
had herbivorous characteristic. It is thus interesting to further characterize whether the subgenome interaction and dominance derive dietary adaptation of this hybrid fish.
\end{abstract}

(Continued on next page)

\footnotetext{
* Correspondence: Isj@hunnu.edu.cn; gzyexing@163.com

†'Wuhui Li, Shi Wang and Jie Hu contributed equally to this work.

${ }^{1}$ State Key Laboratory of Developmental Biology of Freshwater Fish, Hunan Normal University, Changsha 410081, Hunan, China

${ }^{2}$ Key Laboratory of Tropical and Subtropical Fishery Resource Application and Cultivation of Ministry of Agriculture and Rural Affairs, Pearl River Fisheries Research Institute, Chinese Academy of Fishery Sciences, Guangzhou 510380, Guangdong, China
}

(c) The Author(s). 2021 Open Access This article is licensed under a Creative Commons Attribution 4.0 International License, which permits use, sharing, adaptation, distribution and reproduction in any medium or format, as long as you give appropriate credit to the original author(s) and the source, provide a link to the Creative Commons licence, and indicate if changes were made. The images or other third party material in this article are included in the article's Creative Commons licence, unless indicated otherwise in a credit line to the material. If material is not included in the article's Creative Commons licence and your intended use is not permitted by statutory regulation or exceeds the permitted use, you will need to obtain permission directly from the copyright holder. To view a copy of this licence, visit http://creativecommons.org/licenses/by/4.0/ The Creative Commons Public Domain Dedication waiver (http://creativecommons.org/publicdomain/zero/1.0/) applies to the data made available in this article, unless otherwise stated in a credit line to the data. 
(Continued from previous page)

Results: Differential expression, homoeolog expression silencing and bias were investigated in the hybrid fish after 70 days of adaptation to carnivorous and herbivorous diets. A total of $2.65 \times 10^{8}$ clean reads (74.06 Gb) from the liver and intestinal transcriptomes were mapped to the two parent genomes based on specific SNPs. A total of 2538 and 4385 differentially expressed homoeologous genes (DEHs) were identified in the liver and intestinal tissues between the two groups of fish, respectively, and these DEHs were highly enriched in fat digestion and carbon metabolism, amino acid metabolism and steroid biosynthesis. Furthermore, subgenome dominance were observed in tissues, with paternal subgenome was more dominant than maternal subgenome. Moreover, subgenome expression dominance controlled functional pathways in metabolism, disease, cellular processes, environment and genetic information processing during the two dietary adaptation processes. In addition, few but sturdy villi in the intestine, significant fat accumulation and a higher concentration of malondialdehyde in the liver were observed in fish fed carnivorous diet compared with fish fed herbivorous diet.

Conclusions: Our results indicated that diet drives phenotypic and genetic variation, and the asymmetric expression of homoeologous genes (including differential expression, expression silencing and bias) may play key roles in dietary adaptation of hybrid fish. Subgenome expression dominance may contribute to uncovering the mechanistic basis of heterosis and also provide perspectives for fish genetic breeding and application.

Keywords: Allodiploid hybrid, Homoeologous genes, Subgenome expression dominance, Dietary adaption,

\section{Background}

Hybridization can quickly merge two or more divergent genomes to form new allopolyploids and is a driving force of genomic evolution and speciation [1]. The merged subgenomes must adjust and coexist with one another in a single nucleus, frequently causing genetic incompatibilities that induce a series of rapid genetic and epigenetic modifications [2]. These modifications include sequence elimination, activation of genes and retroelements, homoeologous interactions and exchanges, which may directly affect the establishment of a nascent polyploid and its evolutionary success as a new species [3-5]. An observation that may be easily linked to the success of newly formed polyploids is the variations in the expression of homeologous genes, including differential expression and subgenome dominance (one of the parental subgenomes having retained more genes and being more highly expressed, including expression silencing and bias), which have been observed in some allopolyploid plants and a few animals [2-8]. Subgenome dominance contributing to partitioning of certain phenotypic traits has been reported in cotton (Gossypium) [9], wheat (Triticum aestivum) [10, 11], maize (Zea mays ssp. mays L) [12] and blueberry (Vaccinium corymbosum) [13].

In fish, as in other vertebrates, genetic variability and phenotypic plasticity could promote within-species or within-population diversity of individual foraging strategies, while dietary shift (adaption) is likely to enhance the overall phenotypic variance and additive genetic evolution in fish populations [14-17]. Genetic variability, including genome evolution (genetic variation, gene duplication, gene family expansion and contraction, allopolyploidy, etc.) and gene regulation (epigenetic), contributing to feeding behavior and dietary adaptation, have been documented in humans [18], dogs [19], giant panda (Ailuropoda melanoleuca) [20], bats [21] and fishes [7, 22, 23]. Recently, growing evidences has shown that feeding also drives phenotype and genetic evolution, which has been demonstrated in perch (Perca fluviatilis L.) [15], stickleback (Teleostei: Stichaeidae) [24], and baleen whales (Llanocetus denticrenatus) [25]. However, the genotype $\times$ diet interactions contributing to foraging strategies in fish are poorly studied, especially those newly formed hybrid fish species.

Hybridization is an important technique and is widely used in fish genetic breeding. In our previous study, we obtained a hybrid lineage fish derived from herbivorous blunt snout bream (Megalobrama amblycephala, BSB, +) $\times$ carnivorous topmouth culter (Culter alburnus, TC, §) [26]. The hybrid fish are bisexually fertile and contain one set of chromosomes from each parent, providing an idea module to investigate interesting biological processes. A comparative analysis of the gut micorbiota communities and gastrointestinal tract between the hybrid and parents suggested that hybrid fish are biased toward herbivores [27]. A recent study further observed asymmetric allelic expression patterns (including additive and dominant effects and cis and trans regulations) in the hybrid lineages based on the whole-genome sequences of BSB and TC [28]. These results provide new insights into alternative strategies for counteracting deleterious effects of subgenomes and improving the adaptability of novel hybrids. It would thus interesting to further characterize whether the subgenome interaction derives dietary adaptation of the hybrid fish. In this study, we comparatively analyzed the intestinal and liver transcriptomes of hybrid fish after 70 days adaptation to 
herbivorous and carnivorous diets. We focus on the differentially expressed homoeologous genes, as well as the subgenome interaction (homoeolog expression silencing and bias), between the two groups of fish. This study may provide basic data for investigation of the signal transduction pathways that mediate food digestion, metabolism and adaptation and also may provide perspectives for fish breeding and application.

\section{Results}

\section{Histological study of the intestine and liver}

Histological analysis showed a significant difference in the morphology of intestinal and liver tissues in the two dietary groups of hybrid fish. The intestinal villi in fish fed carnivorous diet were sturdy but few in number than those in fish fed herbivorous diet (fine and abundant villi) (Fig. 1a, b). Normal hepatocytes with regular, round nuclei were observed in the livers of both two groups of fish. However, a small amount of hypertrophic hepatocytes lacked nuclei in fish fed herbivorous diet, while a large number of lipid droplets in the liver of the fish fed carnivorous diet were clearly observed (Fig. 1c, d). In addition, significant fat accumulation in the liver tissues of fish fed carnivorous diet was confirmed by Oil red $\mathrm{O}$ staining method (Fig. 1e, f).

\section{Differentially expressed homoeologous genes associated with dietary adaptation}

A total of $2.65 \times 10^{8}$ clean reads $(74.06 \mathrm{~Gb})$ were obtained from the intestinal and liver transcriptomes. Approximately $90.73 \%$ of these clean reads were mapped to the reference genomes based on a total of $2.32 \times 10^{6}$ species-specific SNPs (Table 1, Additional file 1). The complete clean reads were uploaded to the NCBI Sequence Read Archive (SRA) website (https://www. ncbi.nlm.nih.gov/sra/) under accession number PRJNA679638.

Based on the number of mapped reads and genome annotation, DEHs between the two dietary groups of fish were detected (Additional file 2, Table S1). A total of 28, 457 homoeologous genes were coexpressed in the intestine. Among these genes, 4832 DEHs were detected, including 2446 that were more highly expressed and 2386 that were less highly expressed in fish fed herbivorous diet than in fish fed carnivorous diet (Fig. 2a, c). A total of 17,858 genes were coexpressed in the liver, and 2538 DEHs were detected, including 1234 that were more highly expressed and 1304 that were less highly expressed in fish fed with herbivorous diet than in fish fed with carnivorous diet (Fig. 2b, d). The top 24 DEHs in the intestine and liver tissues are presented in Table 2. Compared with fish fed carnivorous diet, some DEHs, such as sqlea, hmgcra, pck1, ulk2 and dio3b, were detected in both intestine and liver tissues in fish fed herbivorous diet.

Specifically expressed genes were detected among different tissues and diets. Seventeen and 11 homoeologous genes were specifically expressed in the liver and intestinal of fish fed herbivorous diet, respectively, and none were specifically expressed in fish fed carnivorous diet (Table S2). In addition, 569 homoeologous genes were specifically expressed in the intestine, and 48 were specifically expressed in the liver, showing no correlation with dietary adaptation (Additional file 3). One homoeologous gene, Rho GTPase-activating protein (arhgap29), was detected in the intestine and liver in only the fish fed herbivorous diet (Additional file 3).

KEGG functional cluster analysis revealed that the intestinal DEHs were enriched in 364 pathways and that the liver DEHs were enriched in 345 pathways. Those DEHs were highly enriched in PPAR signaling pathway (ko03320); biosynthesis of secondary metabolites (ko01110); glycine, serine and threonine metabolism (ko00260); cysteine and methionine metabolism (ko00270); glycerolipid metabolism (ko00561); fat digestion and absorption (ko04975) associated with metabolism in the two dietary groups of fish. The top 15 enriched pathways associated with metabolism in the two dietary groups are presented in Table 3. Interestingly, genes involved in $\mathrm{C} 5$ isoprenoid biosynthesis and mevalonate pathway (Fig. S1) were significantly more highly expressed, and genes involved in the insulin signaling pathway (Fig. S2) were significantly less highly expressed, in both the liver and intestine of fish fed herbivorous diet than those in fish fed carnivorous diet.

\section{Homoeolog expression silencing and bias associated with dietary adaptation}

Based on the mapped read data, subgenome dominance were observed in all tissues, with homoeologous genes derived from parent TC being significantly more highly expressed than those derived from parent BSB. For example, a total of 28,457 homoeolog genes were coexpressed in intestinal tissue, including 6270 annotated to BSB genome, 15,534 annotated to TC genome and 6653 newly assembled genes. In liver tissues, a total of 17,858 genes were coexpressed, including 3312 annotated to BSB genome, 10,699 annotated to TC genome and 3847 newly assembled genes (Fig. 3a, Additional file 2).

Analysis of homoeolog expression silencing (HES) and homoeolog expression bias (HEB) indicated that the TC subgenome was dominant in all tissues (Additional file 4). The amount of BSB-HES was significantly greater than the amount of TC-HES in all tissues, while the amount of TC-HEB was significantly greater than that BSB-HEB $(p<0.05)$, indicating that the homoeologous genes derived from the parent were unbalanced in the hybrid fish 

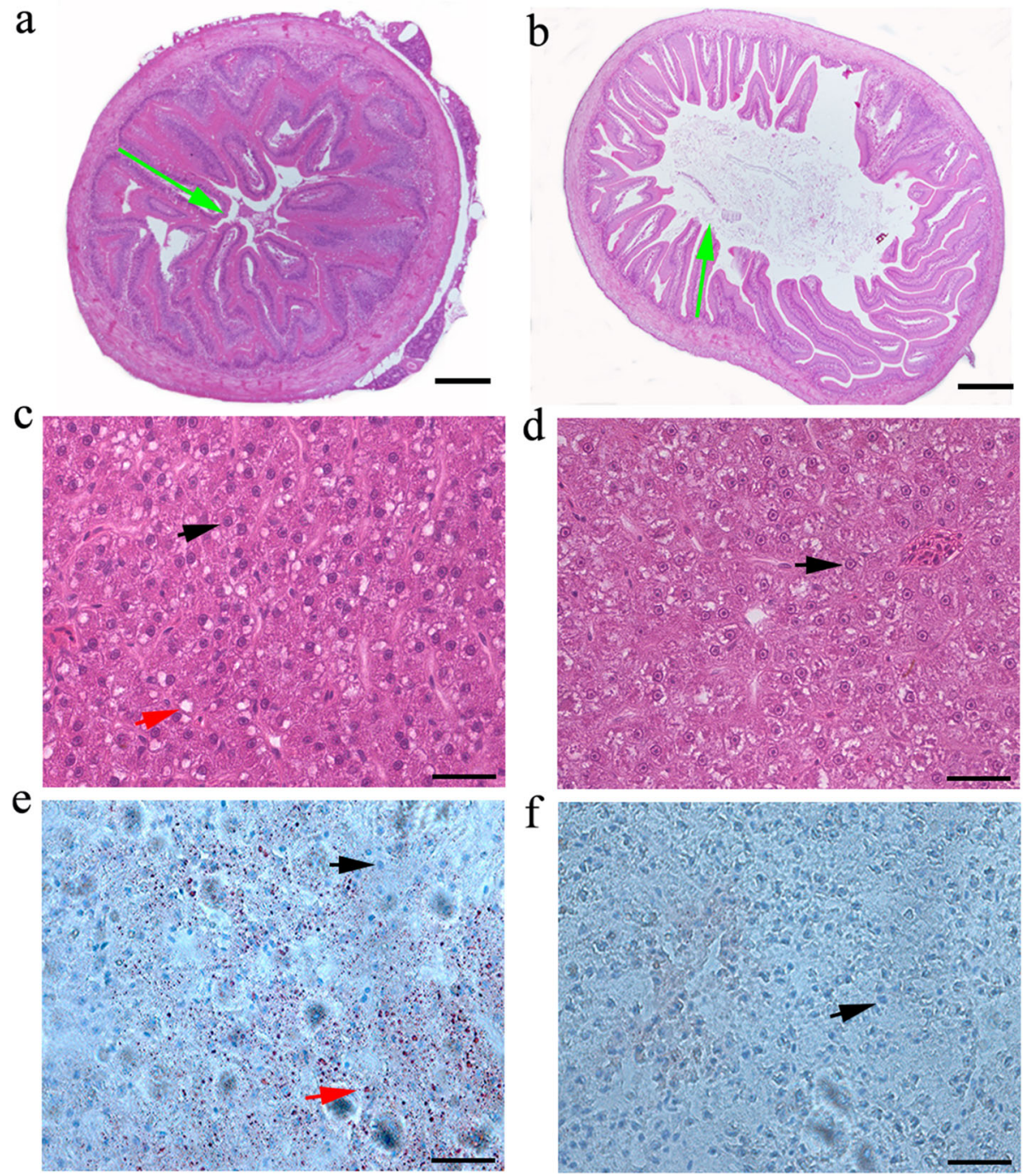

Fig. 1 Representative photomicrographs of the intestinal and liver tissue in hybrid fish fed carnivorous and herbivorous diets. Intestinal tissue from fish fed carnivorous diet a herbivorous diet $\mathbf{b}$. Liver tissue from fish fed carnivorous diet herbivorous diet, stained with H\&E $\mathbf{c}$ and $\mathbf{d}$ and Oil red $\mathrm{O} \mathbf{e}$ and $\mathbf{f}$. The green arrow shows the intestinal villi, the black arrow shows the hepatocytes nucleus, and the red arrow shows the fat cells and lipid droplets

during dietary adaptation (Table 4). Besides, analysis of the shared and specifically expressed homoeologous genes indicated that subgenome dominance was correlated with tissues and almost completely unrelated to diet (Fig. 3b). For instance, the number of homoeologous genes that showed BSB subgenome dominance in the liver and intestine was greater than the number of specially expressed homoeolog genes. The number of homoeolog genes that showed TC subgenome dominance was greater than the number of specifically expressed genes in all tissues (Fig. 3b).

COG functional analysis revealed that the homoeologous genes that showed subgenome dominance were highly enriched in translation ribosomal structure and biogenesis, posttranslational modification, protein turnover, chaperones, carbohydrate transport and metabolism and signal transduction mechanisms (Additional file 5). KEGG functional enrichment analysis revealed that the parent subgenome controlled certain pathways during dietary adaptation. For instance, BSB subgenome mainly controlled metabolism and diseases pathways, such as pyrimidine metabolism, glycosphingolipid biosynthesis, glycosaminoglycan biosynthesis, retinol metabolism, glucosinolate biosynthesis, epithelial cell signaling in Helicobacter pylori infection, and antifolate resistance. The TC subgenome mainly controlled genetic information processing and cellular processes pathways, such as ribosome, spliceosom, aminoacyltRNA biosynthesis, basal transcription factors, ubiquitin mediated proteolysis, peroxisome, lysosome, cell cycle, 
Table 1 Basic information on the intestinal and liver transcriptomes of the two dietary groups of fish

\begin{tabular}{llllll}
\hline Fish group & Total clean reads & Clean reads bases (bp) & SNP Number & Unique mapped reads & Total mapped reads(\%) \\
\hline C-Intestine1 & $40,875,328$ & $6,120,857,148$ & 178,115 & $33,355,355(81.60 \%)$ & $37,659,356(92.13 \%)$ \\
C-Intestine2 & $44,897,254$ & $6,724,235,532$ & 172,520 & $36,725,040(81.80 \%)$ & $41,284,036(91.95 \%)$ \\
C-Intestine3 & $45,432,496$ & $6,801,174,866$ & 176,720 & $36,842,441(81.09 \%)$ & $41,430,875(91.19 \%)$ \\
H-Intestine1 & $45,623,958$ & $6,831,303,448$ & 469,045 & $38,470,050(82.10 \%)$ & $40,048,325(87.78 \%)$ \\
H-Intestine2 & $45,240,496$ & $6,777,495,390$ & 388,932 & $35,867,617(82.10 \%)$ & $40,857,373(90.31 \%)$ \\
H-Intestine3 & $45,129,468$ & $6,760,490,246$ & 296,917 & $32,785,787(82.43 \%)$ & $40,738,675(90.27 \%)$ \\
H-Liver1 & $40,301,740$ & $6,031,302,492$ & 124,038 & $35,510,632(77.83 \%)$ & $37,363,894(92.71 \%)$ \\
H-Liver2 & $50,305,328$ & $7,536,275,016$ & 134,514 & $36,584,989(80.87 \%)$ & $46,520,571(92.48 \%)$ \\
H-Liver3 & $42,994,402$ & $6,436,248,490$ & 113,674 & $36,549,783(80.99 \%)$ & $39,787,469(92.54 \%)$ \\
C-Liver1 & $46,856,834$ & $7,010,976,494$ & 92,877 & $33,170,072(82.30 \%)$ & $43,343,128(92.50 \%)$ \\
C-Liver2 & $43,690,178$ & $6,540,010,734$ & 92,342 & $41,286,506(82.07 \%)$ & $35,867,617(82.10 \%)$ \\
C-Liver3 & $39,773,512$ & $5,952,176,384$ & 87,825 & $35,407,930(82.35 \%)$ & $36,940,518(92.88 \%)$ \\
\hline
\end{tabular}

C-Intestine1 $\sim 3$ and C-Liver1 3: three intestinal and liver tissues of hybrid fish from carnivorous diet group, respectively. H-Intestine1 $~ 3$ and H-Liver1 3: three intestine and liver tissues of hybrid fish from herbivorous diet group, respectively
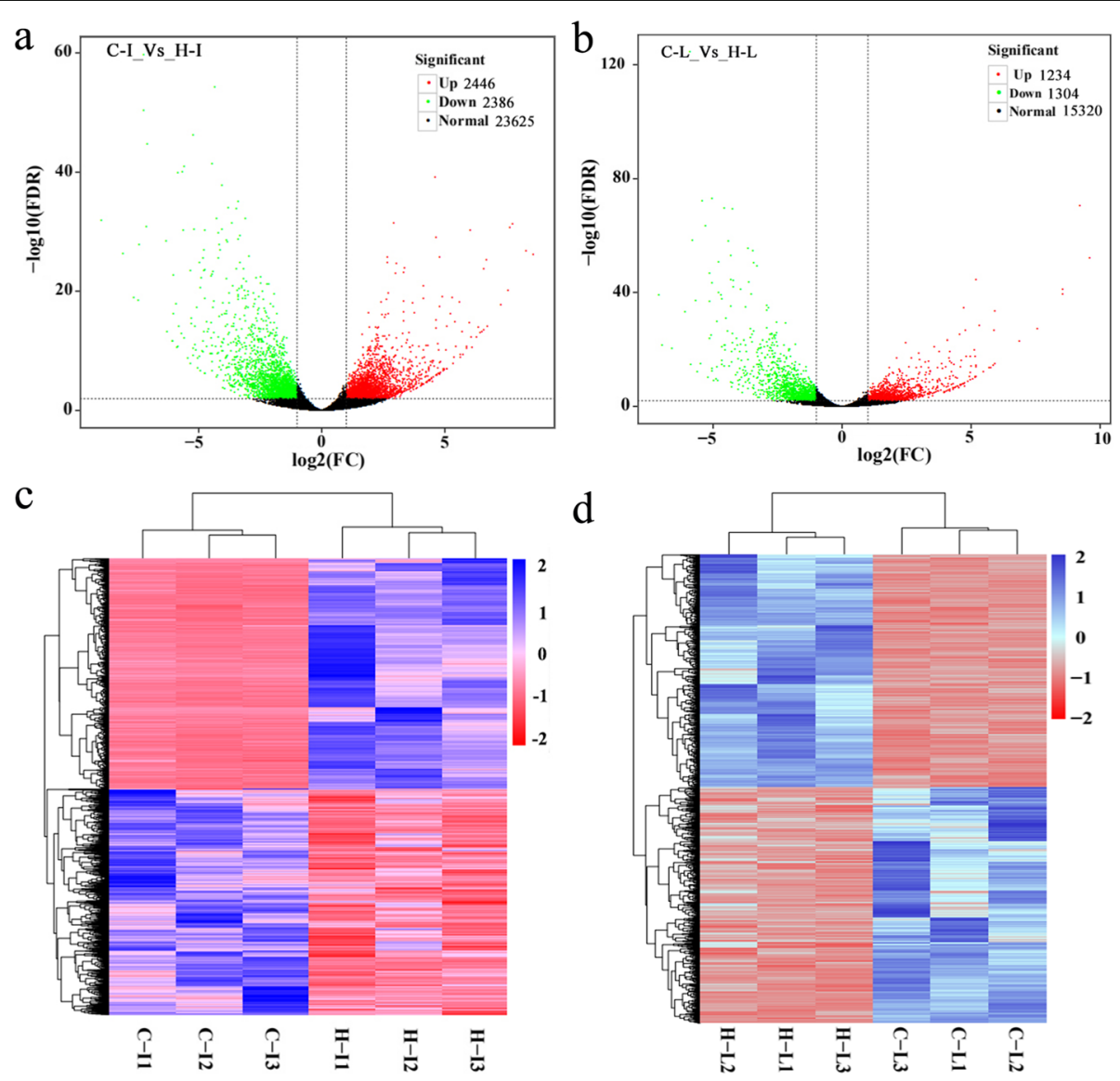

Fig. 2 Cluster analysis of differentially expressed homoeologous genes in intestinal $\mathbf{a}, \mathbf{c}$ and liver $\mathbf{b}$, $\mathbf{d}$ transcriptomes of different dietary groups of fish. The colors and numbers (log10 fold changes) indicate changes in expression levels. C-11 13 and C-L1 L3: intestinal and liver tissues from fish fed carnivorous diet, H-I1 B and H-L1 L3: intestinal and liver tissues from fish fed herbivorous diet 
Table 2 Top 24 differentially expressed homoeologous genes in the intestinal and liver tissues from herbivorous diet group compared with carnivorous diet group

\begin{tabular}{|c|c|c|c|c|c|}
\hline \multicolumn{3}{|c|}{ DEHs in intestine } & \multicolumn{3}{|l|}{ DEHs in liver } \\
\hline Gene symbol & Log2 (fold change) & $\overline{\text { KO ID }}$ & Gene symbol & Log2 (fold change) & KO ID \\
\hline cyp7a1 & 8.30 & K00489 & sqlea & 9.59 & $\bar{K} 00511$ \\
\hline cxcl8b.3 & 7.76 & K10030 & hmgcra & 9.22 & K00021 \\
\hline sqlea & 6.75 & K00511 & cyp51 & 5.91 & K05917 \\
\hline col12aib & 5.64 & K08132 & mcm6 & 5.87 & K02542 \\
\hline fgf4 & 5.59 & K04358 & $n+d 5$ & 5.76 & K17305 \\
\hline rpe65b & 5.40 & K20991 & $a m 2$ & 5.47 & K03910 \\
\hline clca5.2 & 4.98 & K05030 & $\mathrm{mcm} 2$ & 5.20 & K02540 \\
\hline lamc3 & 4.95 & K06247 & epoa & 5.20 & K05437 \\
\hline tubb5 & 4.89 & K07375 & hells & 5.17 & K19001 \\
\hline pppir3ca & 4.79 & K07189 & hsd17b7 & 5.16 & K13373 \\
\hline fuk & 4.76 & K05305 & mcm3 & 5.16 & K02541 \\
\hline hmgcra & 4.73 & K13187 & $e b p$ & 5.12 & K01824 \\
\hline matia & -7.13 & K00789 & $k \mid f 13$ & -5.91 & K09208 \\
\hline diabloa & -7.09 & K10522 & $p c k 1$ & -5.80 & K01596 \\
\hline$p c k 1$ & -6.01 & K01596 & Ipin 1 & -5.30 & K15728 \\
\hline ulk2 & -5.84 & K08269 & lipg & -5.15 & K01046 \\
\hline slc6a19a & -5.79 & K05334 & foxola & -5.05 & K07201 \\
\hline slc26a6 & -5.71 & K14704 & $\arg 2$ & -5.02 & K01476 \\
\hline bcol 1 & -5.28 & K00515 & s/c25a48 & -4.77 & K15124 \\
\hline dio3b & -5.19 & K07754 & tdo2 & -4.69 & K00453 \\
\hline slc15a1b & -4.97 & K14206 & pdzrn3a & -4.66 & K15682 \\
\hline pdegal & -4.63 & K13761 & ulk2 & -4.66 & K08269 \\
\hline uсp 1 & -4.58 & K15103 & $s i k 2 b$ & -4.65 & K16311 \\
\hline ctrl & -4.50 & K09632 & dio3b & -4.53 & K07754 \\
\hline
\end{tabular}

and p53 signaling pathway (Fig. 4, Additional file 5). The relative expression levels of the homoeologous genes associated with food intake, digestion and metabolism, including olfactory transduction, taste transduction, carbon metabolism, starch and sucrose metabolism, fatty acid biosynthesis and protein digestion and absorption are presented in Fig. 5.

\section{qPCR validation}

To validate the quality of the RNA-seq data, we randomly chose $12 \mathrm{DEHs}$ involved in mevalonate pathway and fatty acid biosynthesis pathway (including 3hydroxy-3-methylglutaryl-CoA reductase (hmgcra); glucokinase $(g c k)$; EBP cholestenol delta-isomerase $(e b p)$; isopentenyl-diphosphate delta isomerase 1 (idi1); bytochrome P450 family 51 subfamily A member 1 (cyp51a1), mevalonate diphosphate decarboxylase $(m v d)$; methionine adenosyltransferase 1A (mat1a); lipase G, endothelial type (lipg); phosphoenolpyruvate carboxykinase 1 (pck1); Unc-51-like autophagy activating kinase 1 (ulk1); iodothyronine deiodinase 3 (dio3); beta-carotene oxygenase 1 (bco1)) and performed qPCR with three biological replicates. The relative expression levels of the 12 DEHs are presented in Fig. 6. The trends of the expression levels of these genes detected by qPCR were the same as those obtained by RNA-seq data analysis. These results indicated the reliability of the RNA-seq data for the analysis of differentially expressed homoeoloousg genes during diet adaptation.

\section{Liver biochemical assays}

The concentrations of GSH, MDA, T-SOD and POD in the liver tissue from carnivorous and herbivorous diet fish are presented in Table 5. No significant differences were observed in the GSH, T-SOD and POD between the two dietary groups of fish. However, the concentrations of MDA were significantly higher in fish fed with carnivorous diet than in fish fed with herbivorous diet $(p<0.05, t$-test $)$. 
Table 3 Top 15 enriched pathways associated with differentially expressed homoeologous genes related to metabolism in herbivorous diet group compared with carnivorous diet group

\begin{tabular}{|c|c|c|c|c|}
\hline Pathway in intestine transcriptome & Gene NO. & Reference NO. & $P$ value & Pathway ID \\
\hline Biosynthesis of antibiotics & 109 & 459 & $1.43 \mathrm{E}-13$ & ko01130 \\
\hline PPAR signaling pathway & 40 & 134 & 1.13E-08 & ko03320 \\
\hline Carbon metabolism & 59 & 240 & $1.60 \mathrm{E}-08$ & ko01200 \\
\hline Steroid biosynthesis & 19 & 40 & $2.02 \mathrm{E}-08$ & ko00100 \\
\hline Fat digestion and absorption & 27 & 75 & 3.65E-08 & ko04975 \\
\hline Biosynthesis of secondary metabolites & 138 & 757 & 4.06E-08 & ko01110 \\
\hline Cholesterol metabolism & 33 & 111 & $2.36 \mathrm{E}-07$ & ko04979 \\
\hline Glycine, serine and threonine metabolism & 28 & 86 & $2.39 \mathrm{E}-07$ & ko00260 \\
\hline Cysteine and methionine metabolism & 28 & 87 & $3.14 \mathrm{E}-07$ & ko00270 \\
\hline Biosynthesis of amino acids & 42 & 165 & $6.80 \mathrm{E}-07$ & ko01230 \\
\hline Fatty acid biosynthesis & 15 & 34 & $2.06 \mathrm{E}-06$ & ko00061 \\
\hline Proximal tubule bicarbonate reclamation & 19 & 53 & 4.09E-06 & ko04964 \\
\hline Protein digestion and absorption & 53 & 244 & $5.08 \mathrm{E}-06$ & ko04974 \\
\hline Glycolysis / Gluconeogenesis & 32 & 129 & $2.46 \mathrm{E}-05$ & ko00010 \\
\hline Citrate cycle (TCA cycle) & 19 & 62 & $5.23 \mathrm{E}-05$ & ko00020 \\
\hline Pathway in liver transcriptome & Gene NO. & Reference NO. & $P$ value & Pathway ID \\
\hline Biosynthesis of antibiotics & 80 & 459 & $2.85 E-15$ & ko01130 \\
\hline Metabolic pathways & 257 & 2479 & 8.47E-14 & ko01100 \\
\hline Biosynthesis of secondary metabolites & 103 & 757 & $4.59 \mathrm{E}-12$ & ko01110 \\
\hline Steroid biosynthesis & 16 & 40 & 2.40E-09 & ko00100 \\
\hline Glycine, serine and threonine metabolism & 22 & 86 & $4.08 \mathrm{E}-08$ & ko00260 \\
\hline FoxO signaling pathway & 47 & 303 & 7.83E-08 & ko04068 \\
\hline Insulin signaling pathway & 49 & 323 & 8.44E-08 & ko04910 \\
\hline Cysteine and methionine metabolism & 21 & 87 & $2.42 \mathrm{E}-07$ & ko00270 \\
\hline PPAR signaling pathway & 27 & 134 & $2.68 \mathrm{E}-07$ & ko03320 \\
\hline Cholesterol metabolism & 24 & 111 & $3.14 \mathrm{E}-07$ & ko04979 \\
\hline AMPK signaling pathway & 44 & 298 & $8.28 \mathrm{E}-07$ & ko04152 \\
\hline Terpenoid backbone biosynthesis & 14 & 46 & $1.24 \mathrm{E}-06$ & ko00900 \\
\hline Glyoxylate and dicarboxylate metabolism & 17 & 68 & 1.99E-06 & ko00630 \\
\hline Carbon metabolism & 35 & 240 & $1.43 \mathrm{E}-05$ & ko01200 \\
\hline Adipocytokine signaling pathway & 27 & 169 & 2.64E-05 & ko04920 \\
\hline
\end{tabular}

\section{Discussions}

\section{Diet shifts and adaption affect phenotypic traits}

Fish can change their behavioral and physiological characteristics, such as food selection, food intake, enzyme production, and alimentary tract development, to adjust to changes in the quality of available food [27, 29]. Generally, these adaptive changes are limited by the range of phenotypic plasticity in the short term, and long-term adaptability depends on the evolution of genotype. Previous studies reported that the differences of histological structures among fish gastrointestinal tracts are related to feeding habits, food, age and weight $[30,31]$. In this study, the allodiploid hybrid fish adapted to carnivorous and herbivorous diets also showed a significant difference in histological structures of liver and intestinal tissues (Fig. 1), indicating that food type is associated with the developmental process of gastrointestinal tracts $[14,15]$.

Genetic adaptation to dietary environments is a key process in the evolution of natural populations and is of great interest in fish breeding, as the ability to adaptation to major diet changes can be effectively used to promote fish welfare and a more sustainable aquaculture. Recent studies have focus more on the effect of feeding changes (substituted diets) on growth performance, metabolism, nutrition and gene regulation of fishes [32-34]. 


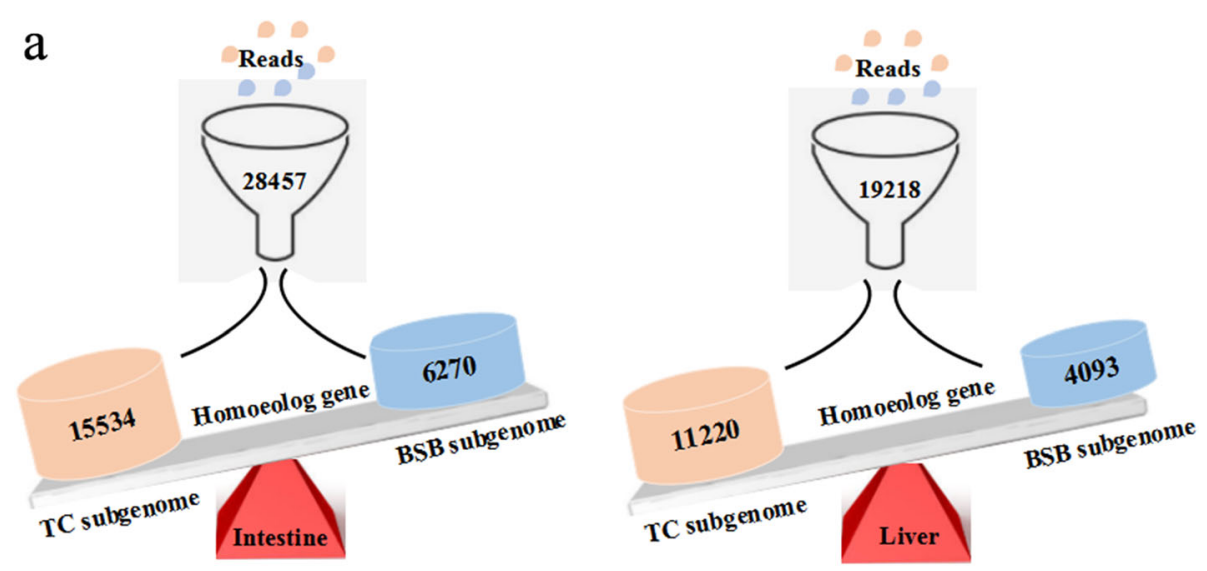

b

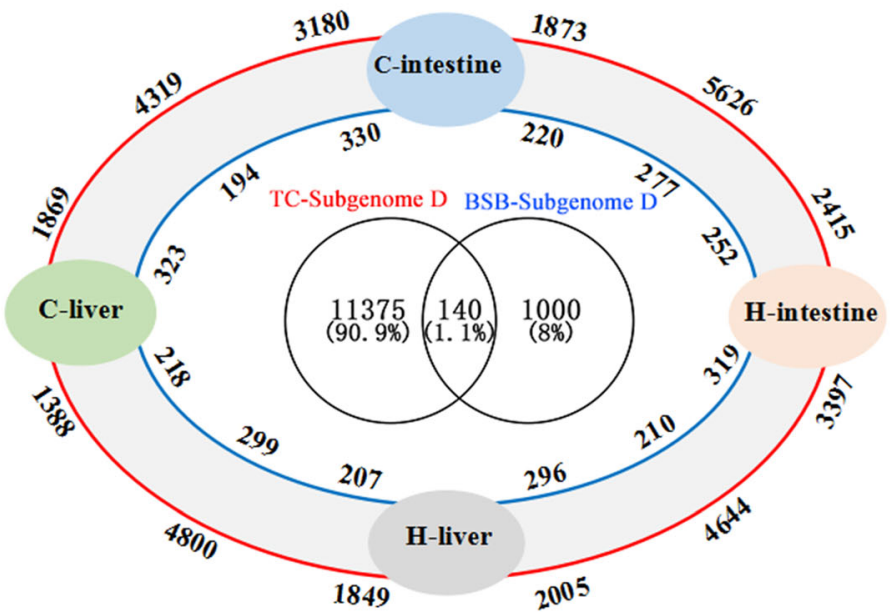

Fig. 3 Subgenome expression dominance in intestinal and liver tissues a and the shared and specially expressed homoeologous genes in different tissues $\mathbf{b}$. The number on the red and blue lines represent the shared and specifically expressed homoeologous genes with TC and BSB subgenome expression dominance, respectively

However, dietary changes and adaptation affect the function of hepatic metabolism to varying degrees, which may result in metabolic and other problems, such as reduced antioxidant capacity, disordered glucose and lipid metabolism, and weakened disease resistance [35-37]. In this study, due to the merging of two subgenome from the carnivorous TC and herbivorous BSB, the hybrid fish seemed to have the potential to adapt to carnivorous or herbivorous diets, and in fact, no impact on growth level was observed in the two dietary groups of fish. In addition, the metabolites related to antioxidant protection, such as GSH, T-SOD and POD, in the liver between the two dietary groups of fish were not significantly different. Higher concentrations of MDA were detected in the carnivorous diet group than in the herbivorous diet group (Table 5), indicating that adaptation to the carnivorous diet more than that to the herbivorous diet affects hepatic metabolism in the hybrid

Table 4 Homoeolog genes expression silencing and bias in tissues from the two dietary groups of fish

\begin{tabular}{|c|c|c|c|c|c|c|}
\hline \multirow[t]{2}{*}{ Type } & \multicolumn{2}{|c|}{ TC subgenome dominance } & \multicolumn{2}{|c|}{ BSB subgenome dominance } & \multirow[t]{2}{*}{ Normal } & \multirow[t]{2}{*}{ Total } \\
\hline & BSB-HES & TC-HEB & TC-HES & BSB-HEB & & \\
\hline C-Intestine & $4522(44.92 \%)$ & $2977(29.57 \%)$ & $243(2.41 \%)$ & $254(2.52 \%)$ & $2070(20.56 \%)$ & 10,066 \\
\hline $\mathrm{H}$-Intestine & $4132(37.17 \%)$ & $3909(35.17 \%)$ & $233(2.10 \%)$ & $296(2.66 \%)$ & $2596(23.35 \%)$ & 11,166 \\
\hline C-Liver & $4482(54.95 \%)$ & 1706 (20.91\%) & $319(3.91 \%)$ & $198(2.43 \%)$ & $1452(17.80 \%)$ & 8157 \\
\hline H-Liver & $4334(49.32 \%)$ & $2315(26.34 \%)$ & 309 (3.52\%) & $197(2.24 \%)$ & $1633(18.58 \%)$ & 8788 \\
\hline
\end{tabular}



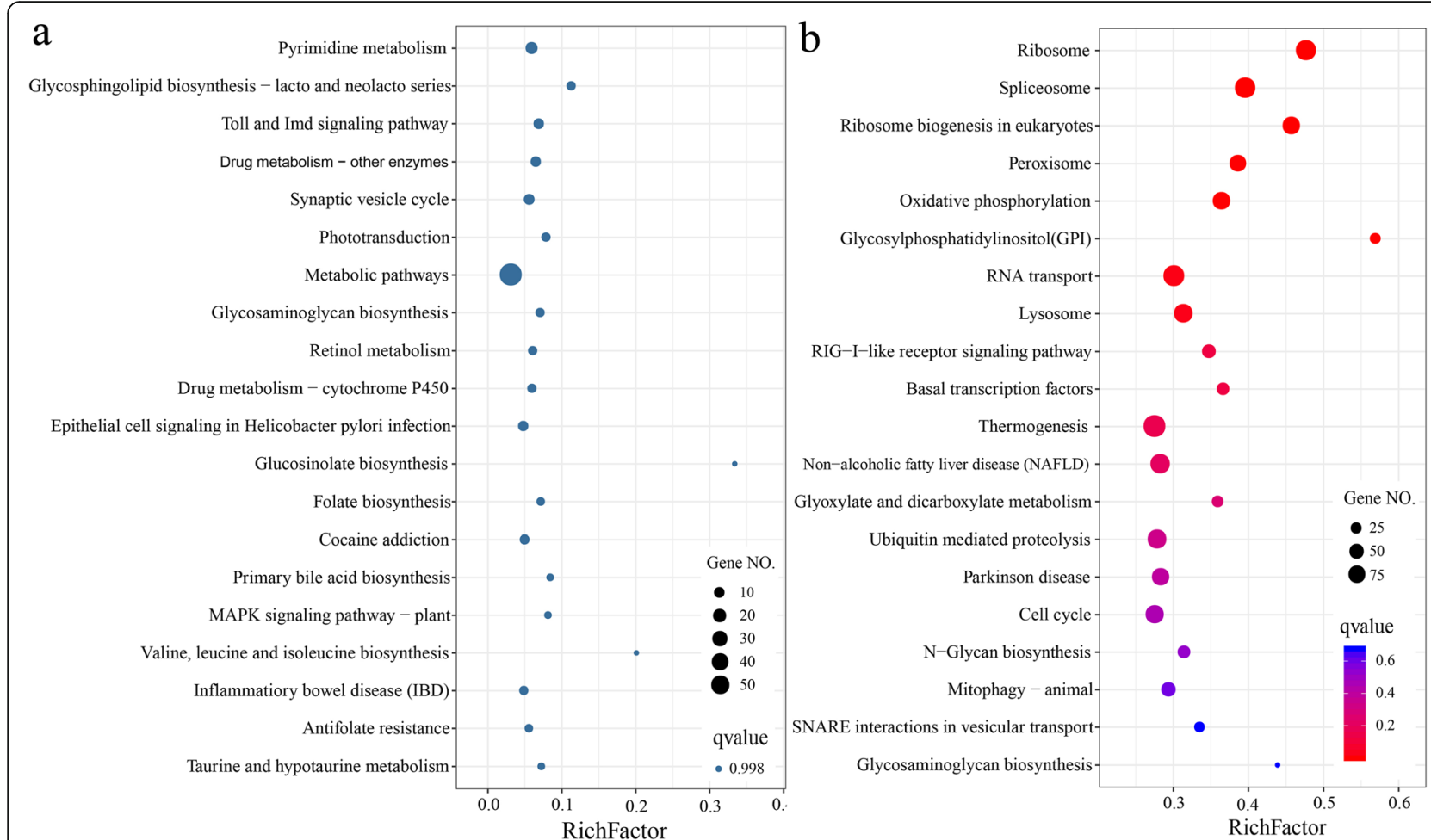

Fig. 4 Top 20 enriched pathways of the expressed homoeologous genes with BSB subgenome expression dominance a and TC subgenome expression dominance $\mathbf{b}$ in the two dietary groups of fish

fish. In addition, the changes in metabolic enzyme activity need to be further studied.

\section{Diet shifts and adaption affect metabolic pathways}

Diet shifts and long-term adaptation can affect major nutrient (carbohydrate, protein and lipid) digestion and metabolism and often lead to significant metabolic changes, such as the modification of certain key metabolic enzymes [35, 38, 39], hepatic metabolic pathways, including energy metabolism, cholesterol biosynthesis and proteolytic activity [35, 38, 40, 41]. Here, we identified a number of DEHs in the hybrid fish adapted to herbivorous diets and carnivorous diets (Fig. 2 and Table 2). These DEHs are highly enriched in carbon metabolism, steroid biosynthesis and amino acid, fatty and protein metabolism (Table 3, Fig. S2 and S3), indicating that diet derives genetic adaption and adjustment to the available food. On the other hand, metabolic adaption of these pathways apparently supports hybrid fish adaptation to carnivorous and herbivorous diets. In European sea bass (Dicentrarchus labrax), genes involved in the LC-PUFA and cholesterol biosynthetic pathways also upregulated when the fish were fed with plantbased diet compared with fish-based diet [42]. In grass carp (Ctenopharyngodon idellus), an herbivorous fish, genes in steroid biosynthesis, terpenoid backbone biosynthesis and glycerophospholipid metabolism pathways were differetinally expressed during carnivorous transition to an herbivorous diet [43]. In BSB, 8 weeks of feeding with a high-fat carbohydrate diet also caused a significant decline in the numbers of amino acids entering TCA cycle, which in turn resulted in elevated levels of seven amino acids [44].

Insulin serves as the primary regulator of blood glucose balance, regulating the metabolism and storage of nutrients such as protein, sugar and fat by acting on liver, muscle and adipose tissue [45]. In BSB, 8 weeks of feeding with a high-carbohydrate diet upregulated the genes associated with insulin signaling pathways, which may lead to the development of insulin resistance in hepatocytes, pathological liver changes, and, eventually, nonalcoholic fatty liver disease [35]. Our study also identified that the insulin receptor, insulin receptorrelated receptor and downstream pathways, including PI (3) K, Akt, FOXO1, Cbl protooncogene and mTOR, were expressed at lower levels in the fish that fed herbivorous diet (Fig. S2), suggesting that the adaptation of hybrid fish to a carnivorous diet may also caused insulin imbalance and insulin resistance in hepatocytes $[35,45]$. 

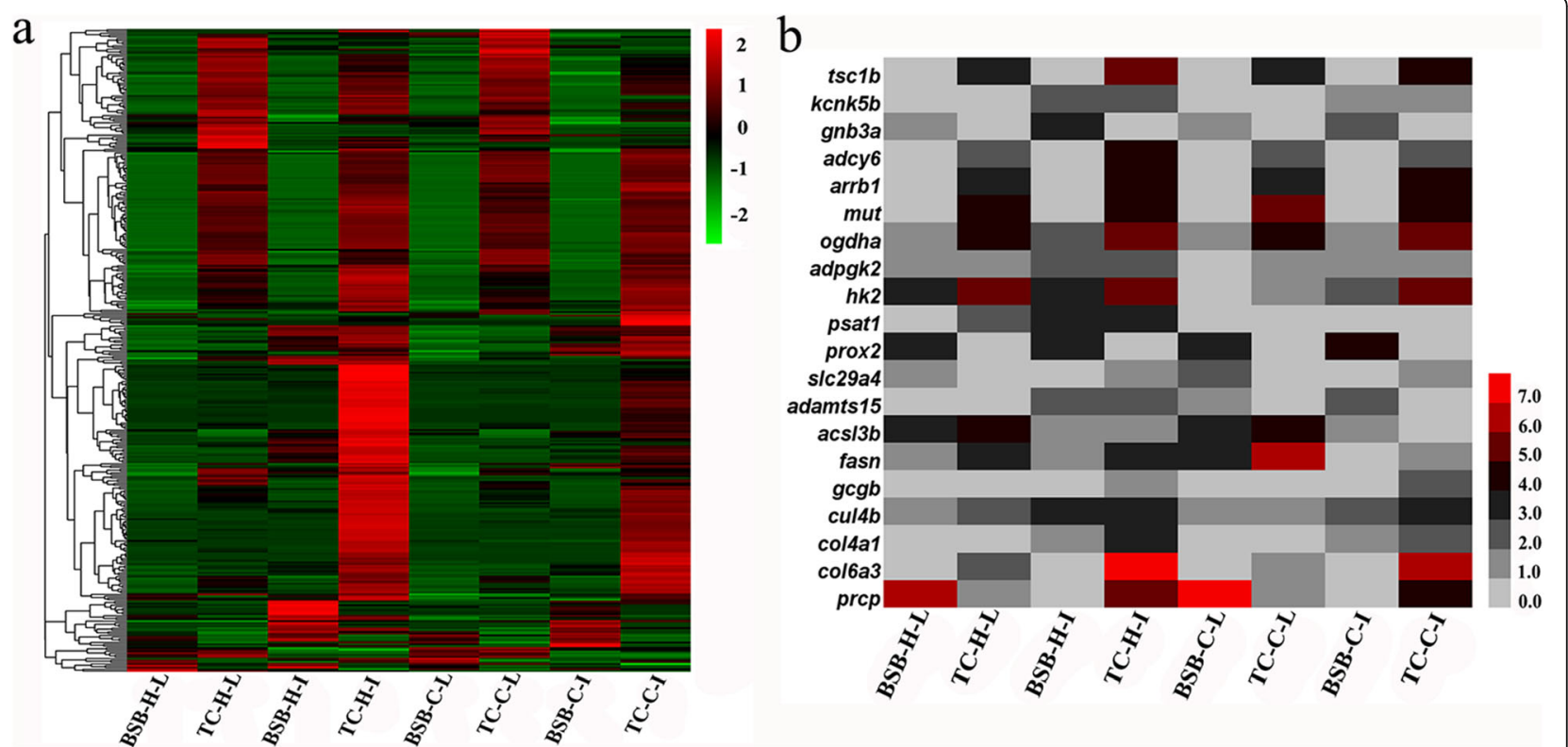

Fig. 5 Relative expression levels of the coexpressed homoeologous genes associated with food intake, digestion and metabolism in the two dietary groups of fish. Global expression level of the coexpressed homoeologous genes involved in taste transduction, olfactory transduction, carbon metabolism, starch and sucrose metabolism, fatty acid biosynthesis and protein digestion and absorption pathways a. Homoeologous genes that showed subgenome expression dominance $\mathbf{b}$. The colors and numbers (log (fold change+ 1, 2)) indicated changes in expression levels. H-L and C-L: represent liver transcriptomes in fish from herbivorous and carnivorous diet groups; $\mathrm{H}-\mathrm{I}$ and C-I: represent intestinal transcriptomes in fish from herbivorous and carnivorous diet groups

\section{Asymmetric expression of homoeologous genes contributes to dietary adaptation}

The merged subgenomes in a polyploid must adjust and coexist with one another in a single nucleus, which may cause interactions between divergent regulatory networks due to differential dosage sensitivity and epigenetic alterations. The variation in the expression of the homoeologous gene is easily observed. To some extent, investigating the variation in the expression of homeolog genes is hindered by at least two major limitations: (1) lack of genomic resources for extant parental progenitors and (2) the inability to distinguish the origins of the subgenome. In our previous study, we completed wholegenome sequencing of the two parents BSB and TC [28], which provided basic date for the present study. Here, subgenome dominance were observed in different tissues, and paternal TC subgenome, more than maternal BSB subgenome dominant expressed during dietary adaptation (Fig. 3). One possible outcome is that the newly formed hybrid must resolve genetic incompatibilities very rapidly within the first generation to adapt environment, including the diet selection and adaptation $[7,9]$. Besides, subgenome expression dominance may be associated with inherent dominance between subgenomes. We also detected the silenced homoeologous genes was unbalanced in tissues, and the silenced homoeologous genes was significantly greater than the bias homoeologous genes during dietary adaptation (Table 4). This may be associated with various regulation elements, such as cis- and trans-regulatory element, lowtransposability elements and DNA methylation [46, 47]. However, the underlying mechanisms for preferential silencing of one parental subgenome over other subgenomes and their functions remain to be further studied.

How one subgenome becomes more highly expressed and whether the direction of subgenome dominance is external induced in a polyploid remain largely unknown. However, a possible scenario in which genetic incompatibilities are resolved and subgenome expression dominance is achieved is when certain subsets of pathways are controlled by one subgenome, while another subgenome controls the remaining set of pathways [11, 48]. Obviously, this would result in the partitioning of phenotypic traits to different subgenomes and is of great significance in genetic breeding of plants and animals. Subgenome dominance contributing to heterosis, such as phenotypic bias, has been observed in several allopolyploid plants [9-12]. In the present study, we also detected parent subgenome controlled functional pathways during dietary adaptation. The BSB subgenome mainly controlled metabolic and diseases pathways, while the TC subgenome mainly controlled genetic information processing and cellular processes (Fig. 4). These pathways, such as the circadian rhythm, digestion and metabolism 

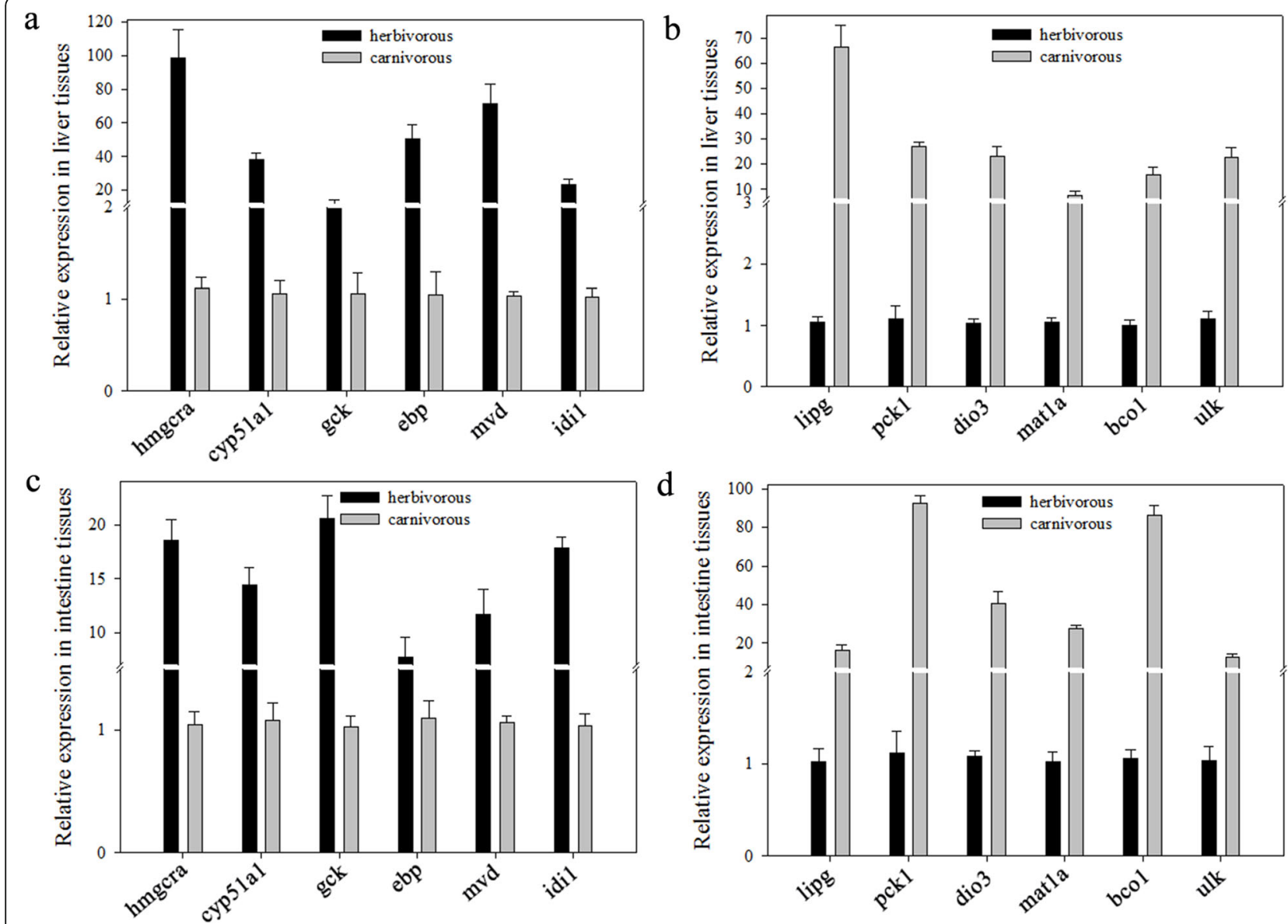

Fig. 6 Real-time polymerase chain reaction analysis of 12 differentially expressed genes between the two dietary groups of fish. Higher and lower expression levels of homoeologous genes in the liver $\mathbf{a}$ and $\mathbf{c}$ and intestine $\mathbf{b}$ and $\mathbf{d}$ of fish fed herbivorous diet compared with fish fed carnivorous diet, respectively. The genes included 3-hydroxy-3-methylglutaryl-CoA reductase (hmgcra), cytochrome P450 family 51 subfamily A member 1 (cyp51a1), glucokinase (gck), EBP cholestenol delta-isomerase (ebp), mevalonate diphosphate decarboxylase (mvd), isopentenyldiphosphate delta isomerase 1 (idi1), lipase G, endothelial type (lipg), phosphoenolpyruvate carboxykinase 1 (pck1), iodothyronine deiodinase 3 (dio3), methionine adenosyltransferase 1A (mat1a), beta-carotene oxygenase 1 (bco1) and Unc-51 like autophagy activating kinase 1 (ulk1)

pathway, were also activated in grass carp during the transition from a carnivorous diet to an herbivorous diet $[43,49]$. Altered expression of pathways involved in retinal photosensitivity, circadian rhythm and appetite control were observed in mandarin fish (Sinipercidae) feed on only live prey fish [50]. Thus, our results indicated that subgenome dominance may play crucial roles in the adaptation of hybrid fish to herbivorous and carnivorous diets. In addition, it would be interesting to investigate whether subgenome expression dominance is heritable and contributes to diet selection in $\mathrm{F}_{2}-\mathrm{F}_{5}$ of this hybrid lineage.

\section{Conclusions}

In summary, the hybrid fish derived from herbivorous BSB (ㅇ) $\times$ carnivorous TC ( $\overbrace{}^{\Uparrow})$ exhibited altered histological structures of liver and intestinal tissues during adaptation to herbivorous and carnivorous diets. Furthermore, homoeologous genes involved metabolism pathways, such as fat, carbon, protein and amino acid metabolism, were differentially expressed during the two types of dietary adaptation. Moreover, subgenome dominance was observed in the hybrid fish, and the direction of expression dominance almost irrelevant to dietary adaptation process. In addition, subgenome expression

Table 5 Biochemical assays of liver tissues in the two dietary groups of fish

\begin{tabular}{lllll}
\hline Type & GSH (g/L) & MDA $(\mathbf{n m o l} / \mathbf{m L})$ & T-SOD $(\mathbf{U} / \mathbf{m L})$ & POD $(\mathbf{U} / \mathbf{m L})$ \\
\hline Carnivorous diet & $50.63 \pm 2.46$ & $182.21 \pm 16.51^{*}$ & $39.23 \pm 0.49$ & $27.18 \pm 0.67$ \\
Herbivorous diet & $59.03 \pm 2.63$ & $4.02 \pm 0.33$ & $41.73 \pm 0.44$ & $23.93 \pm 0.39$ \\
\hline
\end{tabular}

${ }^{*} p<0.05$, representing significant difference between the two groups 
dominance controlled functional pathways during adaptation to herbivorous and carnivorous diets. These results indicated that diet could drive phenotypic and genetic variation, and subgenome expression dominance may play key roles in dietary adaptation in the hybrid fish. This study can provide basic data for the investigation of the signal transduction pathways that mediate food digestion, metabolism and adaption in fish and also may provide perspectives for fish breeding and application.

\section{Methods}

\section{Experimental fish and sampling}

Broodstock BSB and TC were originally obtained from Dong Ting Lake (Hunan province, China). During the reproduction season, from May to June in 2019, sexually matured BSB (female $=5$ ) and TC $($ male $=5)$ were choose as two parents and fish crossing were performed as described previously [26]. Approximately 400 larvae from female $\mathrm{BSB} \times$ male $\mathrm{TC}$ were randomly chosen and reared in two 200-L aquaria. During the breeding process, water temperature was controlled at $25.5 \pm 0.5^{\circ} \mathrm{C}$ under natural photoperiod. The aquaria contained circulating water system, and between 20 and $30 \%$ of the water was replaced every 3 days. The experimental fish fed with Artemia routinely three times a day until 50 days of age. Then, 180 individuals were randomly collected and divided into two group. Each group fish were reared in three $200-\mathrm{L}$ aquaria (each $n=30$ ). One group fish fed Chironomid larvae (defined as carnivorous) and the other fed duckweed and artificial fodder (defined as herbivorous) routinely two times a day. The artificial fodder included the following components (per $100 \mathrm{~g}$ ): fish meal $5.00 \mathrm{~g}$, soybean meal $30.00 \mathrm{~g}$, rapeseed meal $20.00 \mathrm{~g}$, rice bran $35.00 \mathrm{~g}$, fish oil $3.50 \mathrm{~g}$, among others. The amount of food source was gradually increased according to the fish's body weight. The two types of fish $($ each $=6)$ were sampled at 120 days of age after a 12-h fast. The body weights were $21.75 \pm 1.49 \mathrm{~g}$ and $20.11 \pm 0.96 \mathrm{~g}$ for fish fed the carnivorous and herbivorous diet, respectively, and the folk length were $11.63 \pm 0.46 \mathrm{~cm}$ and $11.51 \pm 0.55$ $\mathrm{cm}$. Liver and intestinal tissues were removed, washed with DEPC water and immediately placed into RNALater (Ambion Life Technologies, USA) for mRNA analysis. A piece of liver and intestinal tissue was placed into Bouins' fixative for histological analysis, and the remaining liver tissue was collected and stored in $-70^{\circ} \mathrm{C}$ freezer for biochemical assays.

RNA extraction, library, sequencing and mapping Total RNA from intestine tissues and liver were extracted using TRIzol $^{\odot}$ Reagent, Invitrogen ${ }^{\mathrm{TM}}$ (Life
Technologies) following the manufacturer's instructions. After RNA quality assessment, $1.0 \mu \mathrm{g}$ of total RNA per sample was used as input material for the RNA sample preparations. Sequencing libraries were generated using NEBNext UltraTM RNA Library Prep Kit for Illumina (NEB, USA). First-strand cDNA was synthesized using random hexamer primers and M-MuLV reverse transcriptase. Second-strand cDNA synthesis was subsequently performed using DNA polymerase I and RNase H. After adenylation of 3' ends of DNA fragments, NEBNext adaptors with hairpin loop structures were ligated to prepare for hybridization. The library fragments were purified with AMPure XP system (Beckman Coulter, Beverly, USA) to preferentially select cDNA fragments that were $240 \mathrm{bp}$ in length. Then, $3.0 \mu \mathrm{l}$ of USER Enzyme (NEB, USA) was used with size-selected, adaptor-ligated cDNA at $37^{\circ} \mathrm{C}$ for $15 \mathrm{~min}$ followed by $5 \mathrm{~min}$ at $95^{\circ} \mathrm{C}$ before PCR. Next, PCR was performed with Phusion HighFidelity DNA polymerase, universal PCR primers and index (X) Primer. Finally, PCR products were purified and sequenced on an Illumina HiSeq 2000 platform.

In this study, the genomes of parents BSB $(30,749$ annotated genes) and TC (31,022 annotated genes) from our previous study were used as two reference genomes [28]. After obtaining the paired-end raw reads of each sample, FastQC software (Babraham Bioinformatics) was used to remove reads containing adapter, ploy-N and low quality sequences (unknown nucleotides $>5 \%$ ) [51]. Then, the total clean reads of each sample were aligned to the two reference genomes by using HISAT software based on the species-specific SNPs [52]. The number of mapped reads in each gene was calculated with some inhouse Perl scripts as previously described [28]. Only reads with a perfect match or one mismatch were further analyzed and annotated based on the two reference genomes. Furthermore, to obtain more comprehensive annotation information, StringTie software was used to assemble the mapped reads [52]. After removing the short sequences that encoded peptide chain less than 50 amino acid or contains only a single exon, a total of 11 , 282 new genes were generated, and 9606 can be annotated to at least one databases, including $\mathrm{Nr}, \mathrm{Nt}, \mathrm{Pfam}$, KOG/COG, Swiss-Prot, KO and GO terms.

\section{Differential expression analysis}

Quantification of gene expression levels was estimated by fragments per kilobase of transcript per million fragments mapped (FPKM) method based on the mapped reads [53]. The expression values of three biological replicates were screened with a mean \pm 2 standard deviation (SD) threshold in each gene to avoid interference from expression noise [54]. In addition, the total expression value was normalized based on the ratio of the number of mapped reads for each gene to the total number of 
mapped reads for the entire genome. Finally, DEseq2 in $\mathrm{R}$ software, version 2.13 (R Foundation for Statistical Computing, Vienna, Austria), was used to search for differentially expressed homoeologous genes (DEHs) between the two dietary groups of fish with a false discovery rate $(\mathrm{FDR})<0.01$ and a threshold normalized absolute log 2-fold change $>1.0$ [55]. We also detected the special expressed homoeologous genes between the two dietary groups of fish and different tissues. Specially expressed genes were defined as genes with an expression level of zero (read count $=0$ in three biological replicates) in one tissues or one dietary group of fish (while in the other group or tissues: the read count was $\geqq 3$ in three biological replicates). Gene Ontology (GO) and KEGG pathway analyses were carried out on the free cloud platform BMKCloud (https://international. biocloud.net/zh/user/login) based on the BSB and TC genome annotation.

\section{Analysis of homoeolog expression silencing and bias}

Two sets of genome sequences (annotated protein sequences) were aligned using the reciprocal BLAST (BLASTP, v. 2.2.26) hit method with an e-value cut off of $1 \mathrm{e}^{20}$ to identify orthologs. A total of 20,130 orthologs were obtained for subsequent analysis. Then, the mapped reads of each gene were divided into two categories based on the two different parental reference genomes. BSB homoeolog-specific reads (BSB)/TC homoeolog-specific reads (TC) were used to detect homoeologs expression silencing (HES) and expression bias (HEB), respectively [56]. Expressed genes were defined as BSB silenced (BSB-HES) if $\mathrm{BSB}=0$ and $\mathrm{TC} \geqq 3$ and TC silenced (TC-HES) if BSB $\geqq 3$ and TC $=0$ in the three biological replicates. For analysis of HEB, the expressed genes with $\mathrm{BSB}=0$ and $\mathrm{TC} \leqq 3$ or $\mathrm{BSB} \leqq 3$ and $\mathrm{TC}=0$ were first removed. Then, the expression level of gene was considered biased to BSB subgenome (BSB-HEB) if the ratio of $\mathrm{BSB} / \mathrm{TC} \geqq 2$ and biased to TC subgenome (TC-HEB) if the ratio of $\mathrm{BSB} / \mathrm{TC} \leqq 0.5$. All others cases were considered "normal".

\section{Real-time quantitative PCR (qPCR)}

Total RNA was isolated from liver tissues of the two dietary groups of fish using TRIzol reagent (Invitrogen) according to the manufacturer's instructions. Firststrand cDNA was synthesized using a PrimeScript RT Reagent Kit (RR047A, TAKARA) with PrimeScript RT Enzyme at $37^{\circ} \mathrm{C}$ for $15 \mathrm{~min}$ and at $85^{\circ} \mathrm{C}$ for $5 \mathrm{~s}$ after genomic DNA removal with DNA eraser. qPCR was performed on triplicate technical replicates and ACTN gene (accession no. GU471241) were used as the internal control for normalization of gene expression. qPCR was performed on LightCycler 96 (Roche, Switzerland) and the amplification conditions were as follows: $50^{\circ} \mathrm{C}$ for $5 \mathrm{~min}$,
$95{ }^{\circ} \mathrm{C}$ for $10 \mathrm{~min}$, and 40 cycles at $95^{\circ} \mathrm{C}$ for $15 \mathrm{~s}$ and $60^{\circ} \mathrm{C}$ for $60 \mathrm{~s}$. Then, relative quantification was performed, and melting curve analysis was used to verify the generation of a single product at the end of the assay. The average threshold cycle $(\mathrm{Ct})$ was calculated for each sample using the $2^{-\Delta \Delta \mathrm{Ct}}$ method. The sequences of the primers used are given in Table S3.

\section{Biochemical assays}

Equal amounts of liver tissue from the two dietary groups fish were collected, and homogenates were used to determine the concentrations of superoxide dismutase (SOD), glutataione (GSH), malondialdehyde (MDA) and peroxidase (POD). The assay kits for SOD (A001-1), GSH (A006-1), MDA (A003-1) and POD (A048-2) were purchased from Nanjingjiancheng Bioengineering Institute (Jiangsu, China), and the experimental protocols followed the manufacturer's instructions. The data are presented as the mean \pm standard deviation (SD), and SPSS Statistics 19.0 (IBM Corp., NY, USA) was used to analyze the significance of differences between groups (unpaired two-tailed analysis, $t$ test). The level of statistical significance was set at $p<0.05$.

\section{Abbreviations}

BSB: Megalobrama amblycephala; TC: Culter alburnus; DEHs: Differentially expression homoeologous genes; HES: Homoeolog expression silence; HEB: Homoeolog expression bias.; KEGG: Kyoto Encyclopedia of Genes and Genomes; KO: KEGG orthology; GO: Gene ontology; COG: Cluster of Orthologous Groups of proteins; qPCR: Quantitative real-time PCR; SNPs: Single nucleotide polymorphisms; MDA: Malondialdehyde; SOD: Superoxide dismutase; GSH: Glutataione; POD: Peroxidase

\section{Supplementary Information}

The online version contains supplementary material available at https://doi. org/10.1186/s12864-021-07639-6.

Additional file 1.

Additional file 2.

Additional file 3.

Additional file 4.

Additional file 5 .

Additional file 6: Figure S1. Steroid biosynthesis pathway and the differentilly expressed homoeologous genes in the herbivorous diet group compared with carnivorous diet group. Genes in red boxes were upregulated, and those in green boxes were downregulated.

Additional file 7: Figure S2. Insulin signaling pathway and the differentilly expressed homoeologous genes in the herbivorous diet group compared with carnivorous diet group. Genes in red boxes were upregulated, and those in green boxes were downregulated.

Additional file 8. Supplement tables (Table S1, Table S2 and Table S3).

Acknowledgments

We thank Jun Xiao, Qingfeng Liu, Fangzhou Hu (Hunan Normal University) for their helpful advises on fish crossing and bioinformatics analysis.

\section{Authors'contributions}

This study is conceived and designed by S.J.L. and W.H.L.. Most statistical analyses and write the manuscript by W.H.L, S.W.. Experimental work: C.W., W.H.L., J.H., C.F.S. Experimental materials collect: W.H.L., J.J.D., and J.M.L. 
Manuscript modify: S.J.L. and X.Y.. All authors read and approve the final manuscript.

\section{Funding}

This work was Supported by the National Natural Science Foundation of China (grant Nos. 31702334, 31872315, 31802287), Central Public-interest Scientific Institution Basal Research Fund, CAFS, Grant/Award Number: 2020TD23, the Earmarked Fund for China Agriculture Research System (grant No. CARS-45), and the Open Project Fund of State Key Laboratory of Developmental Biology of Freshwater Fish (2019KF009).

\section{Availability of data and materials}

The complete clean reads were uploaded to the NCBI Sequence Read Archive (SRA) website (https://www.ncbi.nlm.nih.gov/sra/) under accession number PRJNA679638. All data generated or analysed during this study are included in this published article and its supplementary information files.

\section{Declarations}

\section{Ethics approval and consent to participate}

In this study, all experiments were approved by the Animal Care Committee of Hunan Normal University and followed the stated guidelines of the Administration of Affairs Concerning Animal Experimentation of China. Fish collect and crossing were approved by the Animal Care Committee and Protection Station of Polyploidy Fish of Hunan Normal University.

\section{Consent for publication}

Not applicable.

\section{Competing interests}

The authors declare that they have no competing interests.

Received: 19 August 2020 Accepted: 21 April 2021

Published online: 19 May 2021

\section{References}

1. Rieseberg LH, Willis JH. Plant speciation. Science. 2007;317(5840):910-914. https://doi.org/10.1126/science.1137729.

2. Edger PP, Smith RD, Mckain MR, Cooley AM, Vallejomarin M, Yuan Y, et al Subgenome dominance in an interspecific hybrid, synthetic allopolyploid, and a 140-year-old naturally established neo-allopolyploid monkeyflower. Plant Cell. 2017;29(9):2150-67. https://doi.org/10.1105/tpc.17.00010.

3. Jiang B, Lou Q, Wu Z, Zhang W, Wang D, Mbira KG, et al. Retrotransposon- and microsatellite sequence-associated genomic changes in early generations of a newly synthesized allotetraploid Cucumis $\times$ hytivus Chen \& Kirkbride. Plant Mol Biol. 2011;77(3):225-233. https://doi.org/10.1007/s11103-011-9804-y.

4. Wang H, Jiang J, Chen S, Qi X, Fang W, Guan Z, et al. Rapid genetic and epigenetic alterations under intergeneric genomic shock in newly synthesized Chrysanthemum morifolium x Leucanthemum paludosum hybrids (Asteraceae). Genome Biol Evol. 2014;6(1):247-59. https://doi.org/1 0.1093/gbe/evu008.

5. Szadkowski E, Eber F, Huteau V, Lode M, Huneau C, Belcram H, et al. The first meiosis of resynthesized Brassica napus, a genome blender. New Phytol. 2010;186(1):102-12. https://doi.org/10.1111/j.1469-8137.2010.03182.x.

6. Li WH, Liu JM, Tan H, Luo LL, Cui JL, Hu J, et al. Asymmetric expression patterns reveal a strong maternal effect and dosage compensation in polyploid hybrid fish. BMC Genomics. 2018;19(1):517. https://doi.org/10.11 86/s12864-018-4883-7.

7. Liu S, Luo J, Chai J, Ren L, Zhou Y, Huang F, et al. Genomic incompatibilities in the diploid and tetraploid offspring of the goldfish $\times$ common carp cross. PNAS. 2016;113(5):1327-32. https://doi.org/10.1073/pnas.1512955113.

8. Salmon A, Flagel L, Ying B, Udall JA, Wendel JF. Homoeologous nonreciprocal recombination in polyploid cotton. New Phytol. 2010;186(1): 123-34. https://doi.org/10.1111/j.1469-8137.2009.03093.x.

9. Flagel LE, Udall JA, Nettleton D, Wendel JF. Duplicate gene expression in allopolyploid Gossypium reveals two temporally distinct phases of expression evolution. BMC Biol. 2008;6(1):16. https://doi.org/10.1186/17417007-6-16.

10. Eckardt NA. Genome dominance and interaction at the gene expression level in allohexaploid wheat. Plant Cell. 2014;26(5):1834. https://doi.org/10.11 05/tpc.114.127183
11. Pfeifer M, Kugler KG, Sandve SR, Zhan B, Rudi H, Hvidsten TR, et al. Genome interplay in the grain transcriptome of hexaploid bread wheat. Science. 2014;345(6194):1250091. https://doi.org/10.1126/science.1250091.

12. Freeling M, Woodhouse MR, Subramaniam S, Turco GM, Lisch D, Schnable JC. Fractionation mutagenesis and similar consequences of mechanisms removing dispensable or less-expressed DNA in plants. Curr Opin Plant Biol. 2012;15(2):131-9. https://doi.org/10.1016/j.pbi.2012.01.015.

13. Colle M, Leisner CP, Wai CM, Ou S, Bird KA, Wang J, et al. Haplotype-phased genome and evolution of phytonutrient pathways of tetraploid blueberry. GigaScience. 2019;8(3):giz012. https://doi.org/10.1093/gigascience/giz012.

14. Svanback R, Bolnick DI. Intraspecific competition drives increased resource use diversity within a natural population. P Roy Soc B-biol Sci. 2007; 274(1611):839-44. https://doi.org/10.1098/rspb.2006.0198.

15. Svanback R, Persson L. Individual diet specialization, niche width and population dynamics: implications for trophic polymorphisms. J Anim Ecol. 2004;73(5):973-82. https://doi.org/10.1111/j.0021-8790.2004.00868.x.

16. Knudsen R, Klemetsen A, Amundsen P, Hermansen B. Incipient speciation through niche expansion: an example from the Arctic charr in a subarctic lake. P Roy Soc B-biol Sci. 2006;273:2291-8. https://doi.org/10.1098/rspb.2 006.3582 .

17. Dupontnivet M, Medale F, Leonard J, Guillou SL, Tiquet F, Quillet E, et al. Evidence of genotype-diet interactions in the response of rainbow trout (Oncorhynchus mykiss) clones to a diet with or without fishmeal at early growth. Aquaculture. 2009;295(1):15-21. https://doi.org/10.1016/j.aqua culture.2009.06.031.

18. Ye K, Gao F, Wang D, Baryosef O, Keinan A. Dietary adaptation of FADS genes in Europe varied across time and geography. Nat Ecol Evol. 2017;7: 167. https://doi.org/10.1038/s41559-017-0167.

19. Axelsson E, Ratnakumar A, Arendt M, Maqbool K, Webster MT, Perloski M, et al. The genomic signature of dog domestication reveals adaptation to a starch-rich diet. Nature. 2013;495(7441):360-4. https://doi.org/10.1038/na ture11837.

20. Zhao $H$, Yang JR, Xu H, Zhang J. Pseudogenization of the umami taste receptor gene Tas1r1 in the giant panda coincided with its dietary switch to bamboo. Mol Biol Evol. 2010;27(12):2669-73. https://doi.org/10.1093/ meolbev/msq153.

21. Jiao H, Zhang L, Xie HW, Simmons NB, Liu H, Zhao H. Trehalase gene as a molecular signature of dietary diversification in mammals. Mol Biol Evol. 2019;36(10):2171-83. https://doi.org/10.1093/molbev/msz127.

22. Marandel L, Seiliez I, Veron V, Skibacassy S, Panserat S. New insights into the nutritional regulation of gluconeogenesis in carnivorous rainbow trout (Oncorhynchus mykiss): a gene duplication trail. Physiol Genomics. 2015; 47(7):253-63. https://doi.org/10.1152/physiolgenomics.00026.2015.

23. Matthews DG, Albertson RC. Effect of craniofacial genotype on the relationship between morphology and feeding performance in cichlid fishes. Evolution. 2017;71(8):2050-61. https://doi.org/10.1111/evo.13289.

24. German DP, Horn MH. Gut length and mass in herbivorous and carnivorous prickleback fishes (Teleostei: Stichaeidae): ontogenetic, dietary, and phylogenetic effects. Mar Biol. 2006;148(5):1123-34. https://doi.org/10.1007/s00227-005-0149-4.

25. Fordyce RE, Marx FG. Gigantism precedes filter feeding in baleen whale evolution. Curr Biol 2018;28(10):1670-1676.e2. https://doi.org/10.1016/j.cub.2 018.04.027.

26. Xiao J, Kang X, Xie L, Qin Q, He Z, Hu F, et al. The fertility of the hybrid lineage derived from female Megalobrama amblycephala $\times$ male Culter alburnus. Anim Reprod Sci. 2014;151(1):61-70. https://doi.org/10.1016/j.a nireprosci.2014.09.012

27. Li WH, Liu JM, Tan H, Yang CH, Ren L, Liu QF, et al. Genetic effects on the gut microbiota assemblages of hybrid fish from parents with different feeding habits. Front Microbiol. 2018;9:2972. https://doi.org/10.3389/fmicb.2018.02972.

28. Ren L, Li WH, Qin QB, Dai H, Han FM, Xiao J, et al. The subgenomes show asymmetric expression of alleles in hybrid lineages of Megalobrama amblycephala $\times$ Culter alburnus. Genome Res. 2019;29(11):1805-15. https:// doi.org/10.1101/gr.249805.119.

29. Nishio M, Edo K, Kawakami R, Kawamoto T. Morphological changes and habitat shifts with growth of endangered floodplain fish: possible adaptations to fluctuating environments. Ecol Freshw Fish. 2020;29(2):197209. https://doi.org/10.1111/eff.12506.

30. Santos ML, Arantes FP, Pessali TC, Santos JE. Morphological, histological and histochemical analysis of the digestive tract of Trachelyopterusstriatulus (Siluriformes: Auchenipteridae). Zoologia (Curitiba). 2015;32(4):296-305. https://doi.org/10.1590/S1984-46702015000400005. 
31. Gosavi SM, Verma CR, Kharat SS, Pise M, Kumkar P. Structural adequacy of the digestive tract supports dual feeding habit in catfish Pachypterus khavalchor (Siluriformes: Horabagridae). Acta Histochem. 2019;121(4):437-49. https://doi.org/10.1016/j.acthis.2019.03.006

32. Gu Z, Mu H, Shen H, Deng K, Liu D, Yang M, et al. High level of dietary soybean oil affects the glucose and lipid metabolism in large yellow croaker Larimichthys crocea through the insulin-mediated PI3K/AKT signaling pathway. Comp Biochem phys B. 2019;231:34-41. https://doi.org/10.1016/j. cbpb.2018.12.003.

33. Wabike EE, Wu X, Zhu W, Lou B, Chen $R$, Xu D, et al. Partial replacement of fish oil with terrestrial lipid blend and effects on growth performance, body composition, immune parameter and growth-related genes in yellow drum (Nibea albiflora). Aquac Nutr. 2020;26(3):954-63. https://doi.org/10.1111/a nu.13053.

34. Ofori-Mensah S, et al. Fish oil replacement with different vegetable oils in gilthead seabream, Sparus aurata diets: effects on fatty acid metabolism based on whole-body fatty acid balance method and genes expression. Aquaculture. 2020;529:735609. https://doi.org/10.1016/j.aquaculture.2020.73 5609.

35. Prisingkorn W, Prathomya P, Jakovlic I, Liu H, Zhao YH, Wang WM. Transcriptomics, metabolomics and histology indicate that highcarbohydrate diet negatively affects the liver health of blunt snout bream (Megalobrama amblycephala). BMC Genomics. 2017;18(1):856. https://doi. org/10.1186/s12864-017-4246-9

36. Borey M, Paroissin C, Quille E, Terrier F, Maunas P, Burel C, et al. Acute hypoxia reveals diverse adaptation strategies to fully substituted plantbased diet in isogenic lines of the carnivorous rainbow trout. Aquaculture. 2018;490:288-96. https://doi.org/10.1016/j.aquaculture.2018.02.005.

37. Pham HD, Siddik MAB, Fotedar R, Chaklader R. Substituting fishmeal with lupin lupinus angustifolius kernel meal in the diets of cobia Rachycentron canadum: effects on growth performance, nutrient utilization, haematophysiological response, and intestinal health. Anim Feed Sci Tech. 2020;267: 114556. https://doi.org/10.1016/j.anifeedsci.2020.114556.

38. Veron V, Panserat $\mathrm{S}$, Boucher RL, Labbe L, Quillet $\mathrm{E}$, Dupontnivet $\mathrm{M}$, et al. Long-term feeding a plant-based diet devoid of marine ingredients strongly affects certain key metabolic enzymes in the rainbow trout liver. Fish Physiol Biochem. 2016;42(2):771-85. https://doi.org/10.1007/s10695-015-01 74-2.

39. Lee $\mathrm{S}$, Azarm HM, Chang KH. Effects of dietary inclusion of fermented soybean meal on growth, body composition, antioxidant enzyme activity and disease resistance of rockfish (Sebastes schlegeli). Aquaculture. 2016;459: 110-6. https://doi.org/10.1016/j.aquaculture.2016.03.036.

40. Zhang Y, Li Y, Liang X, Cao X, Huang L, Yan J, et al. Hepatic transcriptome analysis and identification of differentially expressed genes response to dietary oxidized fish oil in loach Misgurnus anguillicaudatus. PLoS One. 2017; 12(2):e0172386. https://doi.org/10.1371/journal.pone.0172386.

41. Zhang L, Liu WB, Brown PB, Xu C, Shi HJ, Zheng XC, et al. Utilization of raw and gelatinized starch by blunt snout bream Megalobrama amblycephala as evidenced by the glycolipid metabolism, glucose tolerance and mitochondrial function. Aquaculture. 2020;529:735603. https://doi.org/10.101 6/j.aquaculture.2020.735603.

42. Geay F, Ferraresso S, Zamboninoinfante $J$, Bargelloni L, Quentel C, Vandeputte $M$, et al. Effects of the total replacement of fish-based diet with plant-based diet on the hepatic transcriptome of two European sea bass (Dicentrarchus labrax) half-sibfamilies showing different growth rates with the plant-based diet. BMC Genomics. 2011;12(1):522. https://doi.org/10.11 86/1471-2164-12-522.

43. Wang Y, Lu Y, Zhang Y, Ning Z, Li Y, Zhao Q, et al. The draft genome of the grass carp (Ctenopharyngodon idellus) provides insights into its evolution and vegetarian adaptation. Nat Genet. 2015;47(6):962. https://doi.org/10.103 8/ng0815-962a.

44. Prathomya P, Prisingkorn W, Jakovlic I, Deng FY, Zhao YH, Wang WM. $1 \mathrm{H}$ NMR-based metabolomics approach reveals metabolic alterations in response to dietary imbalances in Megalobrama amblycephala. Metabolomics. 2017;13(2):17. https://doi.org/10.1007/s11306-016-1158-7.

45. Saltiel $A R$, Kahn CR. Insulin signalling and the regulation of glucose and lipid metabolism. Nature. 2001;414(6865):799-806. https://doi.org/10.103 8/414799a.

46. Alger El, Edger PP. One subgenome to rule them all: underlying mechanisms of subgenome dominance. Curr Opin Plant Biol. 2020;54:10813. https://doi.org/10.1016/j.pbi.2020.03.004.
47. Ren L,Yan XJ,Gao X, Cui JL, Yan PC, Wu C, et al. Maternal effects shape the alternative splicing of parental alleles in reciprocal cross hybrids of Megalobrama amblycephala $\times$ Culter alburnus. BMC Genomics. 2020;21(1): 1-12. https://doi.org/10.1186/s12864-020-06866-7.

48. Bird KA, Vanburen R, Puzey JR, Edger PP. The causes and consequences of subgenome dominance in hybrids and recent polyploids. New Phytol. 2018; 220(1):87-93. https://doi.org/10.1111/nph.15256.

49. He S, Liang X, Li L, Sun J, Wen Z, Cheng X, et al. Transcriptome analysis of food habit transition from carnivory to herbivory in a typical vertebrate herbivore, grass carp Ctenopharyngodon idella. BMC Genomics. 2015;16(1): 15. https://doi.org/10.1186/s12864-015-1217-x.

50. He S, Liang X, Sun J, Li L, Yu Y, Huang W, et al. Insights into food preference in hybrid $\mathrm{F} 1$ of Siniperca chuatsi $(+) \times$ Siniperca scherzeri $\left({ }^{\wedge}\right)$ mandarin fish through transcriptome analysis. BMC Genomics. 2013;14(1):601. https://doi. org/10.1186/1471-2164-14-601.

51. Andrews S. FastQC: a quality control tool for high throughput sequence data. 2010.

52. Pertea M, Kim D, Pertea G, Leek JT, Salzberg SL. Transcript-level expression analysis of RNA-seq experiments with HISAT, StringTie and Ballgown. Nat Protoc. 2016;11(9):1650-67. https://doi.org/10.1038/nprot.2016.095.

53. Mortazavi A, Williams BA, Mccue K, Schaeffer L, Wold BJ. Mapping and quantifying mammalian transcriptomes by RNA-Seq. Nat Methods. 2008:5(7): 621-8. https://doi.org/10.1038/nmeth.1226.

54. Quackenbush J. Microarray data normalization and transformation. Nat Genet. 2002;32(\$4):496-501. https://doi.org/10.1038/ng1032.

55. Love Ml, Huber W, Anders S. Moderated estimation of fold change and dispersion for RNA-seq data with DESeq2. Genome Biol. 2014;15(12):550. https://doi.org/10.1186/s13059-014-0550-8.

56. Yoo M, Szadkowski E, Wendel JF, et al. Homoeolog expression bias and expression level dominance in allopolyploid cotton. Heredity. 2013;110(2): 171-80. https://doi.org/10.1038/hdy.2012.94.

\section{Publisher's Note}

Springer Nature remains neutral with regard to jurisdictional claims in published maps and institutional affiliations.
Ready to submit your research? Choose BMC and benefit from:

- fast, convenient online submission

- thorough peer review by experienced researchers in your field

- rapid publication on acceptance

- support for research data, including large and complex data types

- gold Open Access which fosters wider collaboration and increased citations

- maximum visibility for your research: over $100 \mathrm{M}$ website views per year

At $\mathrm{BMC}$, research is always in progress.

Learn more biomedcentral.com/submissions 